\title{
Pulmonary artery diameter to predict pulmonary hypertension in pulmonary sarcoidosis
}

\begin{abstract}
To the Editor:
Pulmonary hypertension $(\mathrm{PH})$ is a known complication of pulmonary sarcoidosis with a prevalence ranging from $5 \%$ to $74 \%$ [1]. The aetiology of $\mathrm{PH}$ in sarcoidosis is not fully understood. Usually, it is attributed to the destruction of the distal capillary bed by lung fibrosis and/or chronic hypoxaemia. However, the severity of PH does not correlate consistently with the degree of pulmonary fibrosis, and PH exists in sarcoidosis patients without fibrosis, suggesting a multifactorial mechanism. The presence of PH is associated with a poor prognosis, and early diagnosis and treatment might improve outcome [1]. Echocardiography should always be performed when $\mathrm{PH}$ is suspected [2]. However, the accuracy of echocardiography in patients with interstitial lung diseases is often limited due to poor image quality and unreliable tricuspid regurgitation signal to measure the right ventricular systolic pressure (RVSP) [3]. Further invasive investigation with the gold standard, right heart catheterisation (RHC), is often required. In order to optimise the noninvasive diagnostic approach, there is a need for more accurate predictors of $\mathrm{PH}$. Computed tomography (CT) may raise suspicion of PH in symptomatic patients or those examined for unrelated indications by showing an increased pulmonary artery (PA) diameter $(\geqslant 29 \mathrm{~mm})$ and PA diameter/ascending aorta diameter (AAD) ratio $(\geqslant 1.0)[2]$. Similarly, PA diameter indexed to body surface area (BSA) has been suggested as possible predictor of $\mathrm{PH}$. However, these parameters have never been investigated in pulmonary sarcoidosis specifically.

In this study, PA diameter measurements on chest CT were retrospectively evaluated as predictors of $\mathrm{PH}$. Patients suspected of PH and referred for analysis between November 2007 and May 2014 were included in cases with a consensus diagnosis of pulmonary sarcoidosis, aged $\geqslant 18$ years, with availability of chest CT within 1 year of PH analysis. The analysis protocol was based on the European PH guideline [2], and consisted of an ECG, laboratory testing, and echocardiographic assessment of RVSP and secondary parameters. Subsequently, patients were classified as "PH likely" (RVSP $>50 \mathrm{mmHg}$ ), "PH possible" (RVSP of 36-50 mmHg or presence of secondary signs with normal/absent RVSP signal) or "PH unlikely" (RVSP $<36 \mathrm{mmHg}$ or absence of signal without secondary signs). RHC was performed if $\mathrm{PH}$ was possible or likely. PH was defined as an invasively measured mean PA pressure (mPAP) $\geqslant 25 \mathrm{mmHg}$. Patients were divided into three groups: "PH confirmed by RHC", "no PH confirmed by RHC" and "no PH based on echocardiography".
\end{abstract}

A radiologist, specialised in interstitial lung diseases and blinded to haemodynamic data, reviewed the chest CT performed most recently to the echocardiographic analysis. The PA diameter was measured at the level of the bifurcation along the line from the centre of the adjacent ascending aorta perpendicular to the main PA axis [4]. As a derivative, the PA diameter was indexed to BSA [5] and calculated as a ratio with AAD. Furthermore, the percentage of fibrosis of the total lung area was described as "not significant" $(<5 \%)$, "intermediate" $(5-20 \%)$ or "severe" $(>20 \%)$.

In total, 89 out of 103 pulmonary sarcoidosis patients referred for $\mathrm{PH}$ analysis were eligible for inclusion. The estimated prevalence of $\mathrm{PH}$ was $28.1 \%$. Table 1 shows the baseline characteristics and outcomes. 16 patients had been referred as a result of a dilated PA. After excluding these patients, PA measurements remained highly significant. PA diameter indexed to BSA and PA diameter/AAD ratio correlated moderately with RHC-derived mPAP ( $\mathrm{r}=0.51$ and $\mathrm{r}=0.52$, respectively).

Receiver operating curve analysis showed a good accuracy for PA diameter indexed to BSA and PA diameter/AAD ratio (area under the curve (AUC) of 0.88 and 0.81 , respectively; $\mathrm{p}<0.001$ ). Optimal cut-off values were calculated for PA diameter $(30.6 \mathrm{~mm})$, PA diameter indexed to BSA $\left(16.02 \mathrm{~mm} \cdot \mathrm{m}^{-2}\right)$ and PA diameter/AAD ratio (1.06). PA diameter indexed to BSA showed the best positive and negative predictive value for diagnosing $\mathrm{PH}$ (70\% and $93.2 \%$, respectively) followed by PA diameter/AAD ratio (64.3\% and $88.5 \%$, respectively).

In a subanalysis, we evaluated patients classified as "PH possible" $(\mathrm{n}=18)$. Only PA diameter indexed to BSA was able to significantly discriminate between the presence and absence of $\mathrm{PH}$ (17.0 versus $14.3 \mathrm{~mm} \cdot \mathrm{m}^{-2}$, respectively; $\mathrm{p}<0.006$ ), with a high diagnostic accuracy (AUC 0.91 ) using a cut-off value of $15.2 \mathrm{~mm} \cdot \mathrm{m}^{-2}$. This was notably higher than PA diameter/AAD ratio (AUC 0.71 ). 
TABLE 1 Baseline characteristics and outcomes

PHRC No PHRC No PHecho

\begin{tabular}{cc}
\multicolumn{2}{c}{$p$-value } \\
\hline $\begin{array}{cc}\text { PHRHC versus } \\
\text { no PHRHC }\end{array}$ & $\begin{array}{c}\text { PHRHC versus no } \\
\text { PHRHC or PHecho }\end{array}$ \\
\hline
\end{tabular}

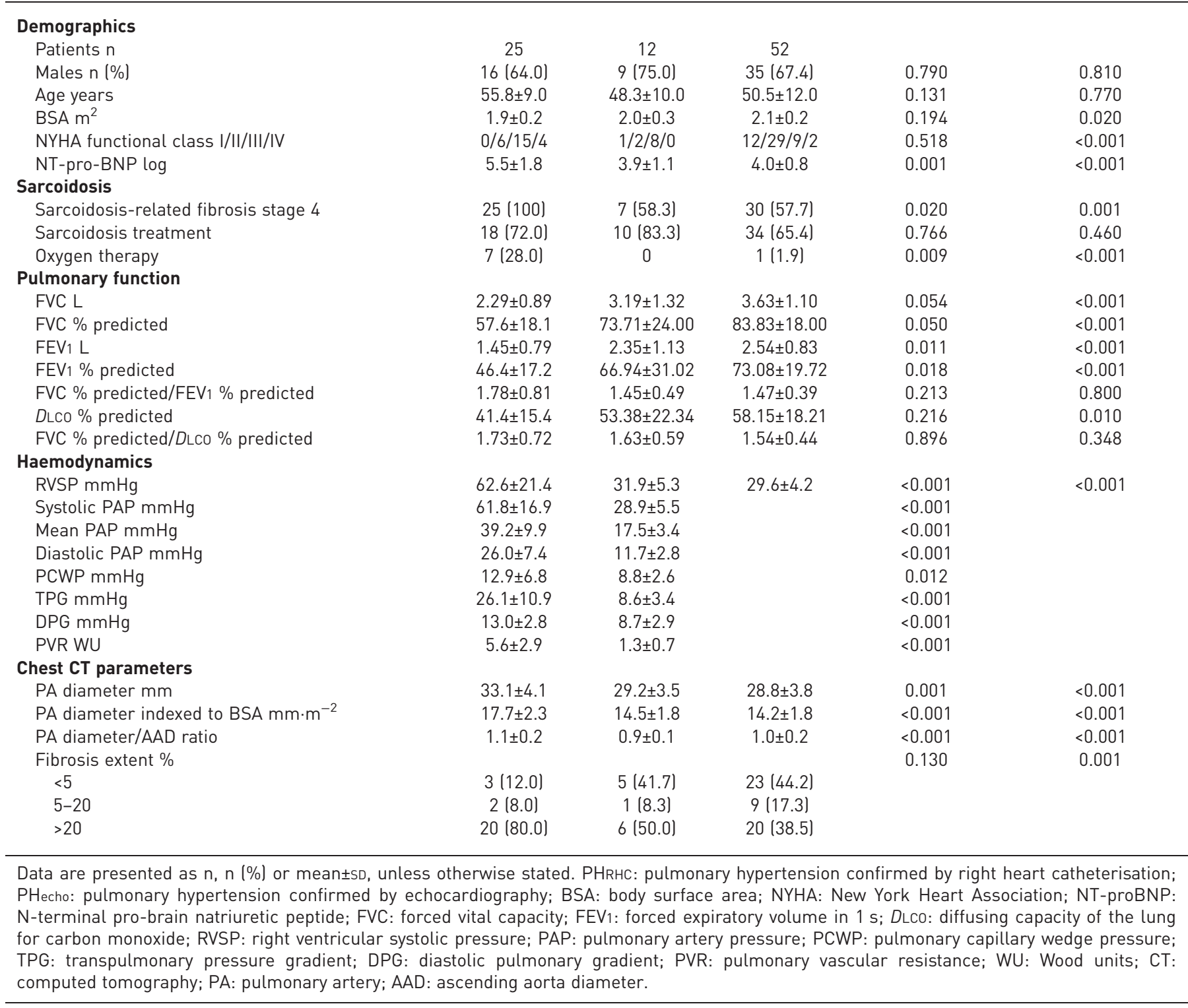

Finally, we evaluated the correlation of PA measurements with the presence of fibrosis. PA diameter and PA diameter indexed to BSA had a weak but significant correlation with the extent of fibrosis $(r=0.32$ and $\mathrm{r}=0.42$, respectively)

This is the first study to investigate chest CT parameters for predicting $\mathrm{PH}$ only in patients with pulmonary sarcoidosis. The results implicate that PA diameter indexed to BSA is the most reliable predictor of $\mathrm{PH}$ for both the general pulmonary sarcoidosis population and for the " $\mathrm{PH}$ possible" subgroup, classified by echocardiography. Therefore, PA measurement on chest CT might be valuable to further optimise $\mathrm{PH}$ analysis in pulmonary sarcoidosis.

Besides hypoxaemia, mechanisms for PH in sarcoidosis include specific vasculopathy of the vessel wall, perivascular fibrosis, external compression of the PA by severe lymphadenopathy, mediastinal fibrosis and left heart disease [1]. PA dilatation in $\mathrm{PH}$ can be due to chronically elevated PA pressure [6]. Similarly to aortic diameter, PA size may vary among individuals, depending on age, sex and BSA [7]. It has been 
suggested that indexing PA to BSA might lead to a higher accuracy that PA diameter/AAD ratio, since BSA influences both PA and aortic diameters [8].

Many studies describe positive correlations for mPAP with PA measurements in different subgroups of $\mathrm{PH}$, especially for PA diameter indexed to BSA and PA diameter/AAD ratio [9]. Only few studies assessed these parameters in interstitial lung diseases, most reporting positive correlations [10-12]. Unfortunately, PA diameter indexed to BSA has not been evaluated in interstitial lung disease. Furthermore, two studies were unable to obtain positive correlations. ZISMAN et al. [13] studied 65 idiopathic pulmonary fibrosis (IPF) patients and found no significant correlation of PA diameters with mPAP, including PA diameter indexed to BSA. However, in that study, a mean mPAP of $26.1 \mathrm{mmHg}$ might have been too low in order to cause dilatation. Similarly, Devaraj et al. [14] found no correlation of mPAP with PA diameter or PA diameter/AAD ratio in 30 patients with pulmonary fibrosis, of whom 11 had sarcoidosis. PA dilatation occurred in the absence of significant $\mathrm{PH}$; however, this was unrelated to the extent of fibrosis. Our study involves the largest group of sarcoidosis patients studied and supports the hypothesis that the mechanism of $\mathrm{PH}$ in sarcoidosis is not fully explained by pulmonary fibrosis. Compared to patients with IPF, pulmonary fibrosis in sarcoidosis is characterised by a chronic course, often involving the upper lobe, and is predominantly of the nonhoneycombing type. Conversely, IPF progresses quickly, is mostly located peripherally or in the lower lobe, with a predominance of honeycombing [15]. This might lead to increased traction on the PA in IPF. For PH in sarcoidosis, the exact mechanism of $\mathrm{PH}$ and $\mathrm{PA}$ dilatation remains unknown, and requires further investigation.

Measurements of PA diameter and AAD are highly reproducible, with an excellent inter- and intraobserver variability [7]. Limitations of this study were mostly due to its retrospective nature, resulting in different types of CT and echocardiography equipment with variable time intervals. Second, not all patients underwent RHC, which might have led to underdetection of $\mathrm{PH}$. Last, this study was conducted in a tertiary care centre.

In conclusion, our study demonstrates that measurement of PA diameter indexed to BSA is the most accurate predictor of $\mathrm{PH}$ on chest $\mathrm{CT}$ in patients with pulmonary sarcoidosis. Prospective research in a large, predefined group of sarcoidosis patients is warranted for further validation and clinical implementation. pulmonary sarcoidosis http://ow.ly/T863m

Marloes P. Huitema ${ }^{1}$, Marcela Spee ${ }^{2}$, Veronique M.M. Vorselaars ${ }^{1}$, Sanne Boerman ${ }^{3}$, Repke J. Snijder ${ }^{3}$, Hendrik W. van $\mathrm{Es}^{2}$, Herre J. Reesink ${ }^{3}$, Jan C. Grutters ${ }^{3,4}$ and Marco C. Post ${ }^{1}$

${ }^{1}$ Dept of Cardiology, St Antonius Hospital, Nieuwegein, The Netherlands. ${ }^{2}$ Dept of Radiology, St Antonius Hospital, Nieuwegein, The Netherlands. ${ }^{3}$ Dept of Pulmonology, St Antonius Hospital, Nieuwegein, The Netherlands. ${ }^{4}$ Division of Heart and Lungs, UMC Utrecht, Utrecht, The Netherlands.

Correspondence: Marloes P. Huitema, St Antonius Hospital Nieuwegein, Koekoekslaan 1, Postbus 2500, 3430 EM Nieuwegein, The Netherlands. E-mail: mp.huitema@antoniusziekenhuis.nl

Received: March 252015 | Accepted after revision: Sept 162015 | First published online: Oct 222015

Conflict of interest: None declared.

\section{References}

1 Seeger W, Adir Y, Barbera JA, et al. Pulmonary hypertension in chronic lung diseases. J Am Coll Cardiol 2013; 62: D109-D116.

2 Galiè N, Humbert M, Vachiery J-L, et al. 2015 ESC/ERS Guidelines for the diagnosis and treatment of pulmonary hypertension. Eur Respir J 2015; 46: 903-975.

3 Fisher MR, Forfia PR, Chamera E, et al. Accuracy of Doppler echocardiography in the hemodynamic assessment of pulmonary hypertension. Am J Respir Crit Care Med 2009; 179: 615-621.

4 Mahammedi A, Oshmyansky A, Hassoun PM, et al. Pulmonary artery measurements in pulmonary hypertension: the role of computed tomography. J Thorac Imaging 2013; 28: 96-103.

5 Du Bois D, Du Bois EF. A formula to estimate the approximate surface area if height and weight be known. 1916. Nutrition 1989; 5: 303-311.

6 Scholzel BE, Post MC, Dymarkowski S, et al. Prediction of outcome after PEA in chronic thromboembolic pulmonary hypertension using indexed pulmonary artery diameter. Eur Respir J 2014; 43: 909-912.

7 Truong QA, Massaro JM, Rogers IS, et al. Reference values for normal pulmonary artery dimensions by noncontrast cardiac computed tomography: the Framingham Heart Study. Circ Cardiovasc Imaging 2012; 5: 147-154.

8 Huitema MP, Reesink HJ, Post MC. Incremental value of pulmonary artery diameter indexed for BSA in pulmonary hypertension. Thorax 2015; 70: 1087.

9 Kuriyama K, Gamsu G, Stern RG, et al. CT-determined pulmonary artery diameters in predicting pulmonary hypertension. Invest Radiol 1984; 19: 16-22.

10 Tan RT, Kuzo R, Goodman LR, et al. Utility of CT scan evaluation for predicting pulmonary hypertension in patients with parenchymal lung disease. Medical College of Wisconsin Lung Transplant Group. Chest 1998; 113: 1250-1256. 
11 McCall RK, Ravenel JG, Nietert PJ, et al. Relationship of main pulmonary artery diameter to pulmonary arterial pressure in scleroderma patients with and without interstitial fibrosis. J Comput Assist Tomogr 2014; 38: 163-168.

12 Alhamad EH, Al-Boukai AA, Al-Kassimi FA, et al. Prediction of pulmonary hypertension in patients with or without interstitial lung disease: reliability of CT findings. Radiology 2011; 260: 875-883.

13 Zisman DA, Karlamangla AS, Ross DJ, et al. High-resolution chest CT findings do not predict the presence of pulmonary hypertension in advanced idiopathic pulmonary fibrosis. Chest 2007; 132: 773-779.

14 Devaraj A, Wells AU, Meister MG, et al. The effect of diffuse pulmonary fibrosis on the reliability of CT signs of pulmonary hypertension. Radiology 2008; 249: 1042-1049.

15 Patterson KC, Hogarth K, Husain AN, et al. The clinical and immunologic features of pulmonary fibrosis in sarcoidosis. Transl Res 2012; 160: 321-331.

\section{Pulmonary hypertension associated with ponatinib therapy}

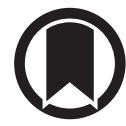

CrossMark

\section{To the Editor:}

Over 50 drugs, including anorexigens, abused substances and recently the tyrosine kinase inhibitor (TKI) dasatinib, have been temporally and/or mechanistically related to the development of pulmonary arterial hypertension (PAH) in humans (where the evidence base for causality is wide ranging) and in animal models [1-3]. The TKIs imatinib, dasatinib, nilotinib and bosutinib are available to treat chronic myelogenous leukaemia (CML) and acute lymphoblastic leukaemia. These drugs are associated with distinctive respiratory adverse effects (AEs), including pleural and/or pericardial effusion, PAH (an AE so far almost exclusively restricted to dasatinib) and pulmonary infiltrates $[1,4-6]$. The novel TKI ponatinib is prescribed for the same indications as above, in patients whose haematological malignancy has become resistant to, or who develop intolerance to the earlier TKIs [7]. Here, we present a female CML patient with prior exposure to dasatinib who developed PAH 6 months into ponatinib therapy, an association that has not previously been reported. Signs and symptoms of PAH improved following drug discontinuation and institution of targeted anti-PAH therapy.

The patient was a Caucasian woman who was diagnosed with CML in 2006, then aged 62 years. Her history included moderate systemic hypertension, dyslipidaemia and obesity (body mass index $38 \mathrm{~kg} \cdot \mathrm{m}^{-2}$ ). She was on long-term treatment with atorvastatin, celiprolol, furosemide, allopurinol, amitriptyline and alprazolam. Treatment for her CML included hydroxycarbamide (500 mg twice daily in July-August, 2006), imatinib (600 mg once daily between July 2006 and October 2008) and nilotinib (400 mg twice daily between October 2008 and January 2011). Dasatinib (100 mg once daily) was started in January 2011. In January 2013, a right-sided lymphocyte-rich (92\%) pleural exudate developed (with proteins $\left.48 \mathrm{~g} \cdot \mathrm{L}^{-1}\right)$. Dasatinib was considered causative and was withdrawn. 1 month afterwards, the effusion was less on imaging and dasatinib was resumed at $100 \mathrm{mg}$ once daily. The effusion relapsed within 6 weeks. Dasatinib was changed to ponatinib (45 mg once daily) in April 2013. Transthoracic echocardiography in May 2013 was normal. N-terminal pro-brain natriuretic peptide (NT-proBNP) was $133 \mathrm{pg} \cdot \mathrm{mL}^{-1}$ (normal is $<167 \mathrm{pg} \cdot \mathrm{mL}^{-1}$ ).

In October 2013, dyspnoea developed, progressing to New York Heart Association (NYHA) class IV. The NT-proBNP was $1500 \mathrm{pg} \cdot \mathrm{mL}^{-1}$. Following a warning from the US Food and Drug Administration on ponatinib-associated arterial and venous thromboses [8], ponatinib was set at $30 \mathrm{mg}$ once daily. Aspirin (100 mg daily) was started. The work-up for dyspnoea included a Doppler ultrasound, which showed right leg vein thrombosis, and fluindione was started. A new echocardiogram disclosed right ventricular dilatation and paradoxical septal motion. Systolic pulmonary artery pressure (PAP) was estimated at $100 \mathrm{mmHg}$. A right-sided catheterisation confirmed severe precapillary pulmonary hypertension with a mean PAP of $52 \mathrm{mmHg}$, normal pulmonary wedge pressure of $12 \mathrm{mmHg}$, low cardiac index $\left(1.8 \mathrm{~L} \cdot \mathrm{min}^{-1} \cdot \mathrm{m}^{-2}\right)$ and elevated pulmonary vascular resistance (12.7 Wood units). There was no acute vasodilator PAP response following inhalation of nitric oxide. An extensive PAH work-up including chest computed tomography (CT) failed to disclose an aetiology for PAH. A ventilation/perfusion scan showed perfectly homogeneous perfusion and ventilation in both lungs. Since no PAH case was known in association with ponatinib at the time, the drug was continued. 